\title{
The SOSTAR-X program achievements
}

\author{
Peter Angenoorth ${ }^{\# 1}$, Luc Chabod ${ }^{* 2}$, Peter Hoogeboom ${ }^{+3}$ \\ \# EADS, Claude Dornier Strasse, 88090 Immenstaad, Germany \\ ${ }^{1}$ peter.angenoorth@eads.com \\ * Thales Airborne Systems 2, av Gay Lussac, 78851 Elancourt, France \\ ${ }^{2}$ luc.chabod@fr.thalesroup.com \\ + TNO Defence, Security and Safety, 2509 JG The Hague, Netherlands \\ ${ }^{3}$ peter.hoogeboom@tno.nl
}

\begin{abstract}
The SOSTAR-X European program started in 2001 and ended in 2007 with successful in-flight tests and operational demonstration. This demonstrator was funded under a $R \& D$ contract based on a governmental MoU between France, Germany, Italy, Spain and The Netherlands. This document presents the scope, content and main results achieved by SOSTAR-X partners.
\end{abstract}

\section{INTRODUCTION}

The major aim of the SOSTAR-X programme was to develop and manufacture a demonstrator for the nextgeneration Imaging/Moving Target Indication active electronically-scanned array antenna radar sensor. The second important goal was its integration into an airborne platform and the demonstration of the feasibility of such a sophisticated system, including a minimum ground element, to satisfy the airborne ground surveillance requirements.

For this purpose, the SOSTAR GmbH Joint Venture Company was established between Dutch Space, EADS, Galileo Avionica, Indra Sistemas and Thales. The company was founded in 2001 to perform the SOSTAR-X Programme.

\section{PROGRAM SCOPE}

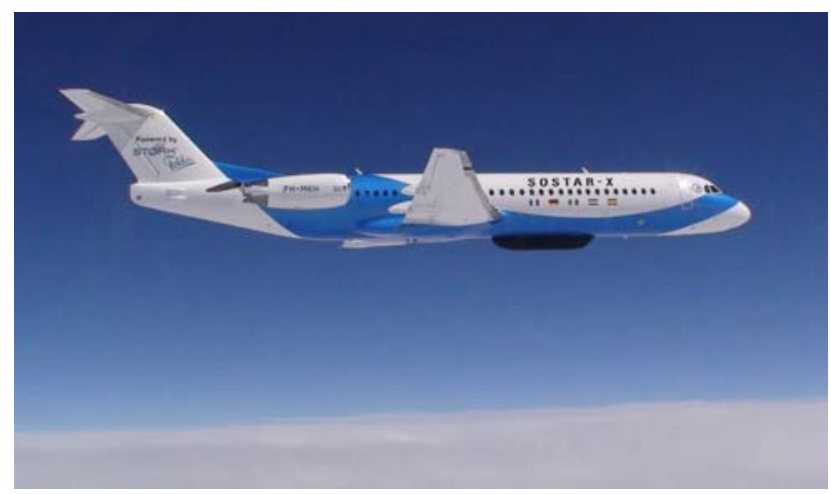

Fig. 1 The SOSTAR system in flight on F-100 (credit : DGA)

Engineering tasks and prime items development (Antenna, Core Electronics, Radar Processor, Operations \& Control) were carried out from 2002 to 2005 .

Then ground integration and aircraft installation were performed up to April 2006. From May 2006 to May 2007, a
Fokker 100, equipped with SOSTAR-X, carried out industrial flights over The Netherlands, Germany and France. Respective support was provided by nations in offering test areas with fixed and moving targets. During these flights, all system modes were tested.

The first part of the industrial flights primarily focussed on optimising the functionalities of the radar sensor and mission management system. The second phase dealt with the progressive integration of real-time processing in the airborne system.

The SOSTAR-X mission was usually pre-planned on the ground however the system provided a realtime inflight replanning capability. The data collected from the missions were recorded for evaluation after flight.

In parallel a data link allowed for imagery to be downlinked in real time to Ground Stations for simultaneous observation on-ground.

In June 2007, the system passed acceptance flights with great success. These flights took place in France to assess the final system performance. The SOSTAR-X system demonstrated extremely high performance across all of its capabilities: Surveillance, Analysis of Target Activity and Classification.

\section{SYSTEM PERFORMANCE}

The SOSTAR-X system demonstrator is dedicated to the surveillance of both fixed and moving targets on large coverage areas, target activity analysis on dedicated areas and classification on designated targets. With this large span of functions, on board operators can gather, monitor and transmit a persistent coverage of the Ground Referenced Coverage Area.

The following paragraphs detail the main system functions and achieved results.

\section{A. Surveillance capability}

First, the SOSTAR surveillance capability provides a SAR stripmap imagery (Synthetic Aperture Radar Area Surveillance Swath - SAR ASS) and a MTI coverage (Moving Target Indication Wide Area Scan - MTI WAS). Both modes were performed in real time and results were displayed onboard the aircraft. 
The greatest challenge for the ASS mode was to provide very large area coverage at long distances. High image quality and accurate localisation were obtained and displayed on board in real time.

The ASS mode proved very high performance levels due to the Strip SAR algorithms developed, with specific Motion Compensation and Autofocus techniques.

The behaviour of this radar mode has been calibrated on the basis of specific test scenarios deployed, showing successful demonstration of Radar Image quality metrics, as well as location accuracy measured on Digital Terrain maps.

Full compliance with all technical requirements was met for all range and squint angle conditions.

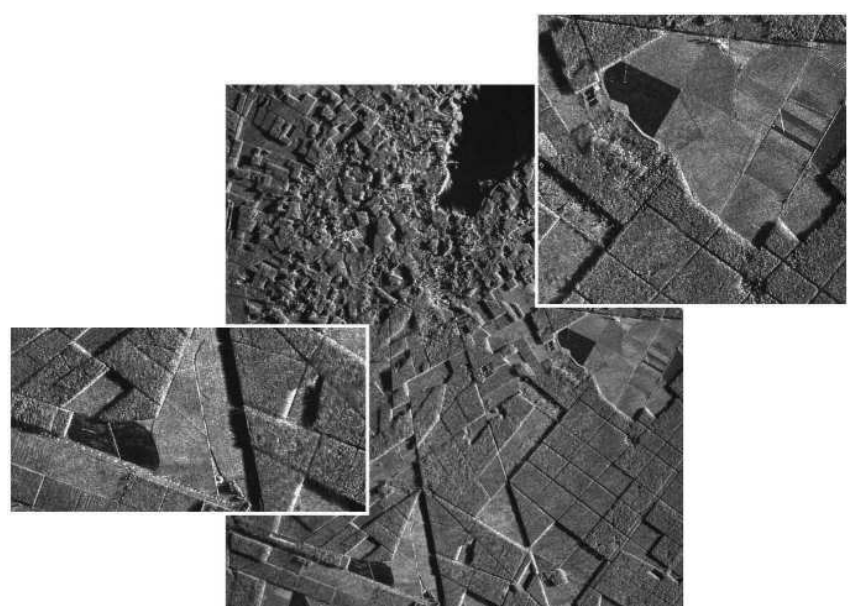

Fig. 2 ASS mode results

The greatest challenge for the MTI WAS mode was to detect at far distances small vehicles moving at speeds comparable with a fast walking man. In order to achieve this Minimum Detectable Velocity (MDV), Space Time Adaptive Processing (STAP) techniques were implemented which today represent the state of the art for real time MTI processing.

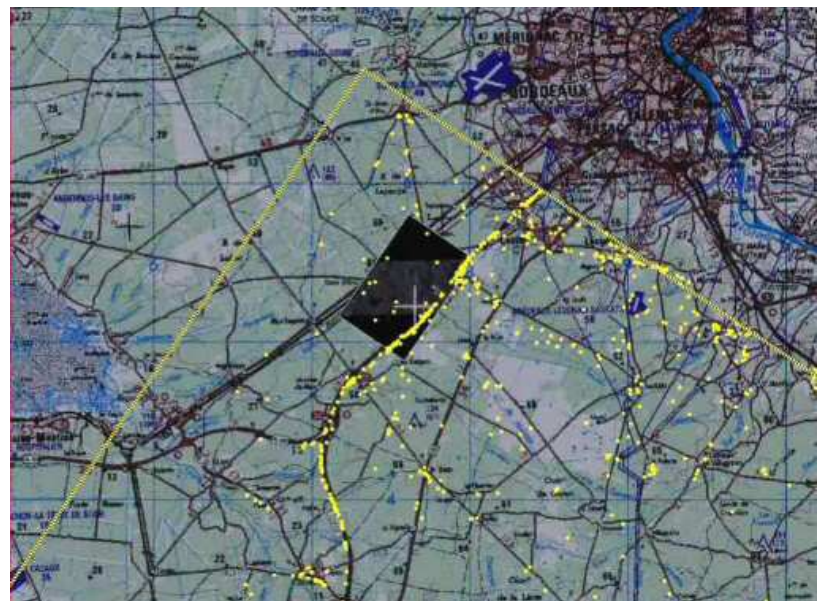

Fig. 3 MTI WAS mode with SAR overlay

The MTI WAS was tested over the whole spread of required distances using many cooperating targets (ground vehicles and helicopters) operating at different speeds, ranging from the MDV to the velocities typical of a low altitude flying aircraft.

Each cooperating vehicle was equipped with a GPS to provide data for efficient post flight analysis. The main performance parameters, like the detection range capability and the target localisation accuracy, resulted compliant with the technical specification within the whole distance coverage.

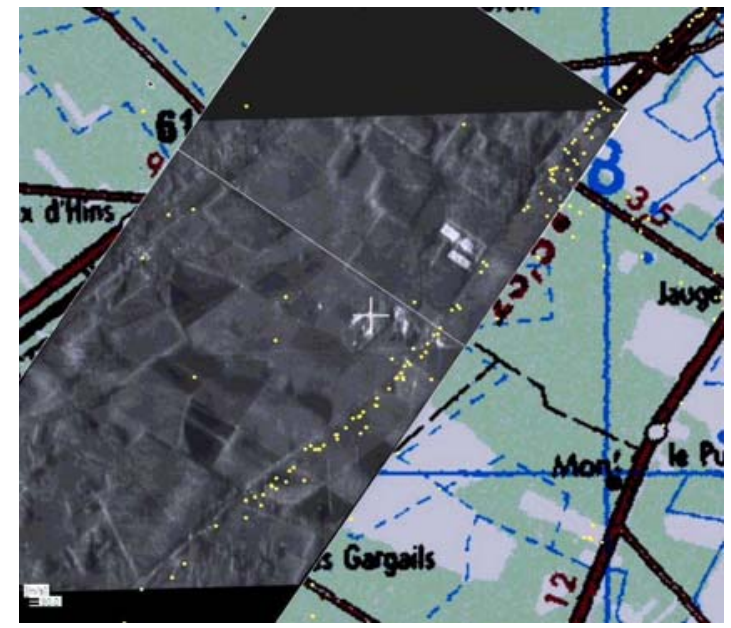

Fig. 4 Zoom on overlaid MTI and SAR results

\section{B. Target activity analysis capability}

Then, the SOSTAR Target Activity capability provides SAR Spot Imagery (Medium and Long Range SAR MRS/LRS), and MTI Fast Sector Scan (MTI FSS) associated with continuous target Tracking Function.

The greatest challenge for the MRS/LRS SAR mode was to deliver images in real time with very high resolution at long distance and in squinted conditions. Image quality and location accuracy are adequate for interpretation purposes.

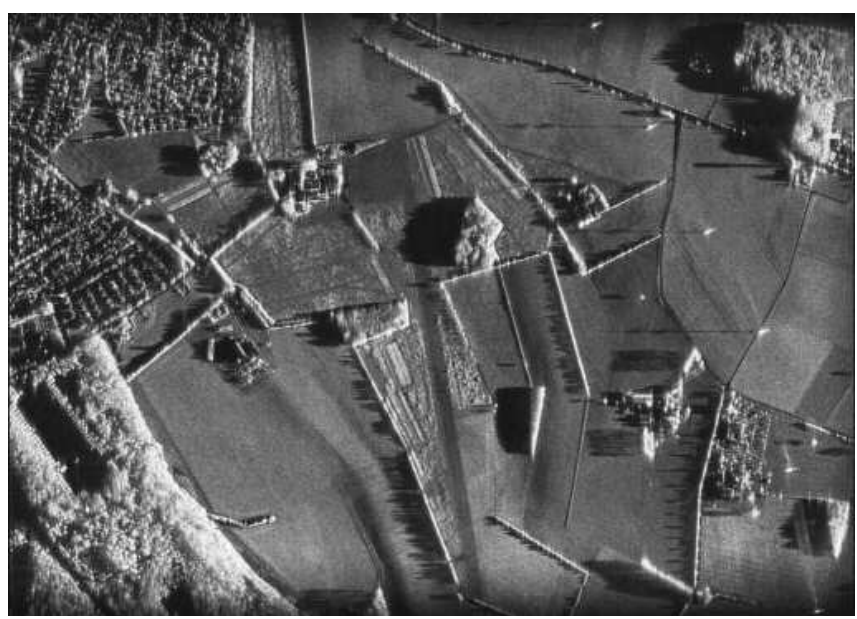

Fig. 5 Spot SAR results

The same corner reflectors were used both for ASS and MRS/LRS to measure the SAR performance parameters. The MRS/LRS mode was tested under full range and squint conditions. 
The real time performance was met and images were displayed on board the aircraft. A first level of analysis was successfully performed with inflight map overlay and zooming for visual interpretation. The main performance parameters like Spot size area, average geometric resolution, peak side lobe ratio and integrated side lobe ratio in azimuth and range resulted compliant with the specification.

The greatest challenge for the MTI FSS mode was to detect slow and fast moving targets within a relatively limited area with a revisit time higher than the MTI WAS. The detected targets were processed by the real-time tracker function in order to provide targets velocity and trajectory estimates. This MTI mode, which uses STAP real-time techniques, was fully tested over several kinds of scenarios (including single ground moving vehicles, convoys and helicopters) specifically tailored on the Tracker operational needs, demonstrating to meet the performance requirements.

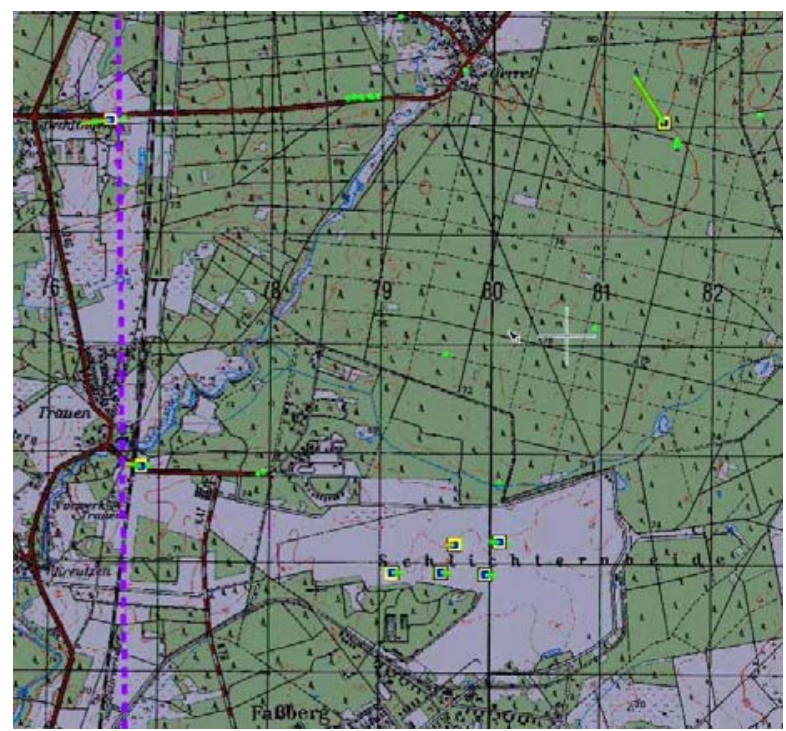

Fig. 6 MTI FSS Mode with Tracker (five wheeled vehicle on a runway and one helicopter)

One of the highest achievements of the mission system is the operation of the Simultaneous Mode, which gives the capability to perform MRS/LRS and MTI/FSS at the same time. This unique mode takes benefit of the high performance active electronic beam steering antenna, which can create simultaneous beams in several directions. Speaking in operational terms, the system is providing detailed analysis of a dedicated spot area and simultaneous detection and tracking of moving target activity in another area. This mode was successfully demonstrated: the quality of the simultaneous antenna beams was verified and the high-quality Spot SAR images and MTI targets were provided in real-time to the onboard operators.

\section{Classification capability}

Finally, a Classification capability based on the spectral Doppler response of target features was developed and demonstrated successfully with test targets. With this mode, the on-board operators designate a track for classification. This track is then illuminated with specific waveforms, response is processed in real-time and the classification results (Wheeled, Tracked, or Helicopter) are provided to the onboard operators. This capability has been demonstrated for the first time in a European airborne radar.
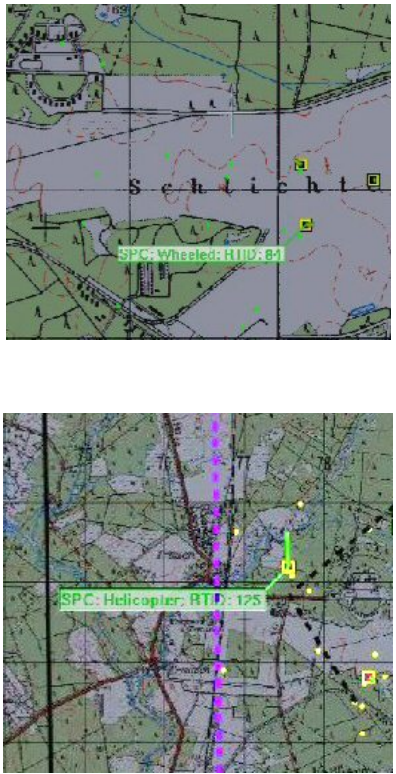

Fig. 7 Wheeled and Helicopter classification results

\section{CONCLUSIONS}

SOSTAR has structured a strong European industrial consortium and an organization of the nations involved, setting the basis for further European cooperation programmes. The SOSTAR programme has provided the participating companies with in-depth knowledge how to design, build, test and assess an airborne ground surveillance system

\section{ACKNOWLEDGMENT}

SOSTAR-X team wishes to acknowledge the five European Governments which have funded SOSTAR-X program, actively participated to the system development and test and therefore enabled significant progress in the air-to-ground surveillance domain. 